\title{
Propuesta de Red de Acceso Óptico para el Centro Histórico de Manzanillo, Cuba: instrumento de estudios para universitarios del sector de las telecomunicaciones
}

\section{Red de Acceso Óptico}

Randy Verdecia Peña, Ing. $\left({ }^{1}\right)$

Manleys Rodríguez Torres, Ing. $\left({ }^{2}\right)$

Dilber Rosabal Montero, Ing. $\left(^{3}\right)$

Leonel Figueredo Fernández, Ing. $\left({ }^{4}\right)$

( $\left.{ }^{1}\right)$ Empresa de Telecomunicaciones de Cuba S.A (ETECSA), Granma, Cuba

$\left({ }^{2}\right)$ Empresa de Telecomunicaciones de Cuba S.A (ETECSA), Granma, Cuba

(3) Universidad de Granma, Granma, Cuba

(4) Universidad de Granma, Granma, Cuba

Contacto: randy.verdecia@etecsa.cu

Receptado: 12/09/2016

Aceptado: 22/11/2016

\section{Resumen}

La formación de ingenieros en el sector de las Telecomunicaciones en Cuba es un desafío para los educandos encargados de llevar adelante este proceso. La carencia de poder demostrar de forma práctica la utilización de la fibra óptica en las redes de accesos motivó a la realización de este proyecto. Para la investigación se tomó como campo de acción, el Centro Histórico de Manzanillo, Granma. El objetivo del trabajo fue proporcionar un instrumento para los estudiantes de esta rama. Se emplearon varios métodos que permitieron la profundización práctica. El instrumento consiste en una propuesta de red de acceso óptico para el Centro Histórico de Manzanillo. Esta aprovecha el terminal de línea óptica existente en el Centro Telefónico de la ciudad. Se valida la propuesta mediante el cálculo del enlace óptico en el que se consideran las pérdidas introducidas por los componentes de la red de distribución óptica. Se concluye que la propuesta garantiza el acceso a los servicios de banda ancha y permite la profundización de los contenidos con mayor eficacia y de forma práctica.

Palabras claves: instrumento de estudio, fibra óptica, enlace óptico, profundización de los contenidos 


\title{
Proposal for Optical Access Network To The Historic Center of Manzanillo, Cuba: Tools for University Studies Telecommunications Sector
}

\begin{abstract}
The formation of engineers on the field of communication in Cuba is a challenge for the teachers in charge of this process. The lack of a tool for having a practical demonstration in networks of the optic fiber was the reason of the project. The Historical center of Manzanillo City in Granmma Province was taken as the research field. The objective of the work was to give an instrument for students on this specialty. There were used several methods which permitted a practical deepening. The tool consisted in a proposal ofa network of optic access in Historical Center of the city. This takes advantage of optic terminal of line existing in the Telephone center of the city. The proposal was valued through the linking optic calculus, in which are considered the loses introduced by the components of the optic distribution network. It was concluded that the proposal makes possible the access to the services of band width, and this permits the students to deepen in the contents more efficiently an in a practical way.
\end{abstract}

Keywords: study tool, optic fiber, optical link, deepening the contents

\section{Introducción}

El acceso juega un papel importante desde el punto de vista tecnológico dentro del desarrollo del modelo de redes. El avance de las tecnologías de acceso debe facilitar el despliegue de nuevas redes y servicios; tal es el caso de la banda ancha, a través de la cual se entregan de manera fiable servicios convergentes y se ofrecen simultáneamente voz, video y datos (UIT, 2013).

La comisión de banda ancha para el desarrollo digital, creada por la Unión Internacional de Telecomunicaciones (ITU, International Telecommunication Union), considera que los países deben usar estrategias y políticas para que la banda ancha sea costeable y esté disponible como herramienta vital para el avance sostenido de la sociedad moderna del conocimiento. Su uso en la vida diaria permitirá el acceso a los servicios de educación, 
Rev. SINAPSIS, Edición № 9, Vol. 2, Diciembre 2016

cultura, entretenimientos, salud, comerciales y financieros en formato digital (ITU-T, 2014a).

Las tecnologías de acceso de banda ancha, en su comienzo, estuvieron soportadas sobre el par de cobre, para la transmisión de voz y datos sobre la red telefónica pública conmutada (PSTN, Public Switched Telephone Network). Las líneas de abonado digital (DSL, Digital Subscriber Line) posibilitaron la extensión de la vida útil de la red de cobre y todavía tienen gran predominio para soportar la banda ancha.

Existen diferentes tipos de redes xDSL, entre ellas ADSL, ADSL2+, VDSL y VDSL2, son las que permiten una mayor tasa de transferencia y se han desarrollado mediante el empleo de técnicas de aceleración de DSL como vectoring y phantom (Zhao, Fischer, Aker, \& Rigby, 2013). Cada una posee características específicas en cuanto al ancho de banda utilizado, velocidad en sentido de descarga y subida, alcance máximo y las aplicaciones que soportan. Es importante destacar que ADSL2+ se ha desplegado ampliamente en la red de acceso y hoy en día es reemplazado por VDSL2 la cual ofrece una mayor tasa de transferencia en ambos sentidos de la comunicación (Lafata, 2013). También, las xDSL han evolucionado hacia G.fast, un nuevo estándar que permite alcanzar velocidades de 1 Gbps sobre el par de cobre.

Además, se han desarrollado las redes ópticas las cuales eliminan el cuello de botella en las redes de acceso, aumentan el flujo de información hasta cientos de gigabit por segundos (Gbps) y mejoran la calidad de servicio. En la actualidad existen varios organismos internacionales, como el FTTH Council, que promueven el despliegue de fibra óptica hasta el hogar o sus inmediaciones, mediante redes FTTx, las cuales se pueden clasificar como: fibra hasta el hogar (FTTH, Fiber to the Home), fibra hasta el edificio (FTTB, Fiber to the Building), fibra hasta la acera (FTTC, Fiber to the Curb), entre otras (FTTH Council Europe, 2012).

Las soluciones tecnológicas existentes también pueden utilizarse en dependencia de la tecnología de acceso empleada y el uso de elementos activos entre la central telefónica y el equipo del usuario final: red óptica pasiva y activa (Koonen, 2006; Mahloo, 2015). La red óptica activa (AON, Active Optical Network) utiliza elementos activos en el tramo de red entre la central telefónica y el cliente. Estos equipos convierten las señales 
Rev. SINAPSIS, Edición № 9, Vol. 2, Diciembre 2016

luminosas que viajan a través de la fibra óptica a señales eléctricas para su procesamiento.

Por su parte, la red óptica pasiva (PON, Passive Optical Network) está compuesta por elementos pasivos para llevar la información hasta el usuario final; el equipamiento activo solo se encuentra en los extremos de la red, por lo cual constituyen una opción factible para su despliegue en la red de acceso. Está compuesta por 3 partes principales (Ansari \& Zhang, 2013): terminal de línea óptica (OLT, Optical Line Terminal), unidad de red óptica (ONU, Optical Network Unit) y la red de distribución óptica (ODN, Optical Distribution Network) correspondiente a cada puerto PON de la OLT. En esta última se localizan los divisores ópticos pasivos (POS, Passive Optical Splitter) encargados de dividir la señal óptica hacia cada usuario.

Entre las PON, una de las tecnologías de mayor aceptación, elegida por varios operadores de telecomunicaciones y fabricantes en el mundo es la red óptica pasiva con capacidad de gigabit (GPON, Gigabit-capable Passive Optical Network), descrita en el estándar ITU-T G.984.1 (ITU-T, 2008).

Como parte del desarrollo de la informatización de la sociedad en Cuba y a partir del programa "Conectar 2020" de la Unión Internacional de Telecomunicaciones (UIT) (ITU-T, 2014b), se demanda a ETECSA (Empresa de Telecomunicaciones de Cuba S.A.) el despliegue de soluciones de acceso de banda ancha para las redes priorizadas de la salud y la educación, así como para redes vinculadas a la producción de software, centros científicos, universidades y hogares. Todo ello es posible con la modernización de la planta externa (OSP, OutSide Plant).

El Centro Histórico de la Ciudad de Manzanillo provincia de Granma cuenta con 2159 viviendas, donde viven 6527 habitantes, con 128 demandas insatisfechas y 785 servicios telefónicos básicos instalados, 12 por cada 100 habitante; 46 públicos, 7 servicios por cada 100 habitantes; incluyendo 172 entidades, con 12 demandas insatisfechas y 423 servicios telefónico básico en el sector no residencial. En este sentido, la Dirección Territorial ETECSA Granma ha realizado estudios en la red existente en diferentes sitios de la provincia con el objetivo de realizar proyectos que permitan satisfacer los requerimientos de crecimiento y velocidad de transmisión de 
Rev. SINAPSIS, Edición № 9, Vol. 2, Diciembre 2016

variados servicios de banda ancha (Carballo-González, Conde del Oso, \& AníasCalderón, 2016).

La investigación partió tomando como problema la carencia de un instrumento que pudiera demostrar de forma práctica los conocimientos adquiridos por los futuros ingenieros en el sector de las telecomunicaciones en Cuba para la implementación de una red de acceso óptica. El Centro Histórico de Manzanillo, Cuba sirvió como campo investigativo donde se realizó el proyecto.

Se expuso en observación previa, que una red óptica para el acceso a banda ancha tanto para el sector residencial como el estatal eliminaría una de las principales problemáticas, garantizando internet en los hogares y satisfaciendo la demanda insatisfecha existente en la región. Además, la misma se utilizada como material de estudio para estudiantes universitarios de la carrera de Telecomunicaciones.

El objetivo del trabajo fue proporcionar mediante la implementación de una red de acceso óptica para el Centro Histórico de Manzanillo, Cuba un instrumento práctico para los futuros ingenieros en el sector de las Telecomunicaciones en Cuba.

\section{Materiales y métodos}

En la investigación se utilizaron métodos teóricos y empíricos. Entre los métodos teóricos se empleó el análisis y síntesis, para la caracterización de los elementos que conforman la red de acceso, así como el estudio de los referentes teóricos sobre el desarrollo de las tecnologías surgidas para garantizar las velocidades que requieren los servicios de banda ancha soportados sobre par de cobre y fibra óptica. La inducción y deducción fueron de utilidad para la elección de la tecnología de acceso que ofrece una solución para la modernización de la planta externa de la red de acceso en la región analizada.

Como método empírico se empleó la observación científica para diagnosticar la situación existente con la demanda de servicios de telecomunicaciones y la red de planta externa en el área objeto de estudio. Se efectuó la entrevista como técnica de recopilación de información para precisar el proceso de diseño de la red en la región. Se determinaron las tecnologías de banda ancha que se pudieran implementar y cuáles se 
Rev. SINAPSIS, Edición № 9, Vol. 2, Diciembre 2016

han desplegado por ETECSA en la red de acceso de sus clientes de acuerdo a las políticas de la empresa.

El desarrollo de la red de acceso es una tarea que necesita de un efectivo diseño de la topología de la red que se pretende desplegar. Para el planeamiento de la red se tuvo en consideración la demanda de servicios de los clientes residenciales y no residenciales, la localización de la ruta que sigue la traza aérea y soterrada, soportada sobre fibra óptica y cobre, así como las características del equipamiento en explotación, su ubicación y la necesidad de su modernización para satisfacer las demandas de servicios en el área de estudio. Esta información permitió la elaboración de la propuesta de red de acceso óptica con la tecnología GPON, con la reutilización de la localización de la terminal de línea óptica en el Centro Telefónico de Manzanillo, la red de postes y de cables de par de cobre existentes en el área.

\section{Resultados}

Como problema investigativo se analizó la inexistencia de una red de acceso óptico en el Centro Histórico de Manzanillo y la ascendente demanda insatisfecha existente en la zona. Además, los estudiantes universitarios de la carrera de Telecomunicaciones y ramas afines en Cuba, no cuentan con un material práctico que le posibilite la implementación de una red de acceso óptica.

Primeramente, se procedió a realizar una caracterización de todo el equipamiento y planta exterior existente en el centro, donde los cables de cobre instalados en el área, en general, son de 200 pares. Los terminales asociados a los cables anteriores se ubican en la mayoría de las intersecciones de calles, son de 20 pares con hasta 15 pares en múltiple. Los bajantes se disponen en largas corridas, que generalmente se cruzan entre terminales a consecuencia de que estos últimos han sido instalados en demanda en una red saturada con más de 30 años de explotación como se muestra en la figura 1. 


\section{Rev. SINAPSIS, Edición № 9, Vol. 2, Diciembre 2016}

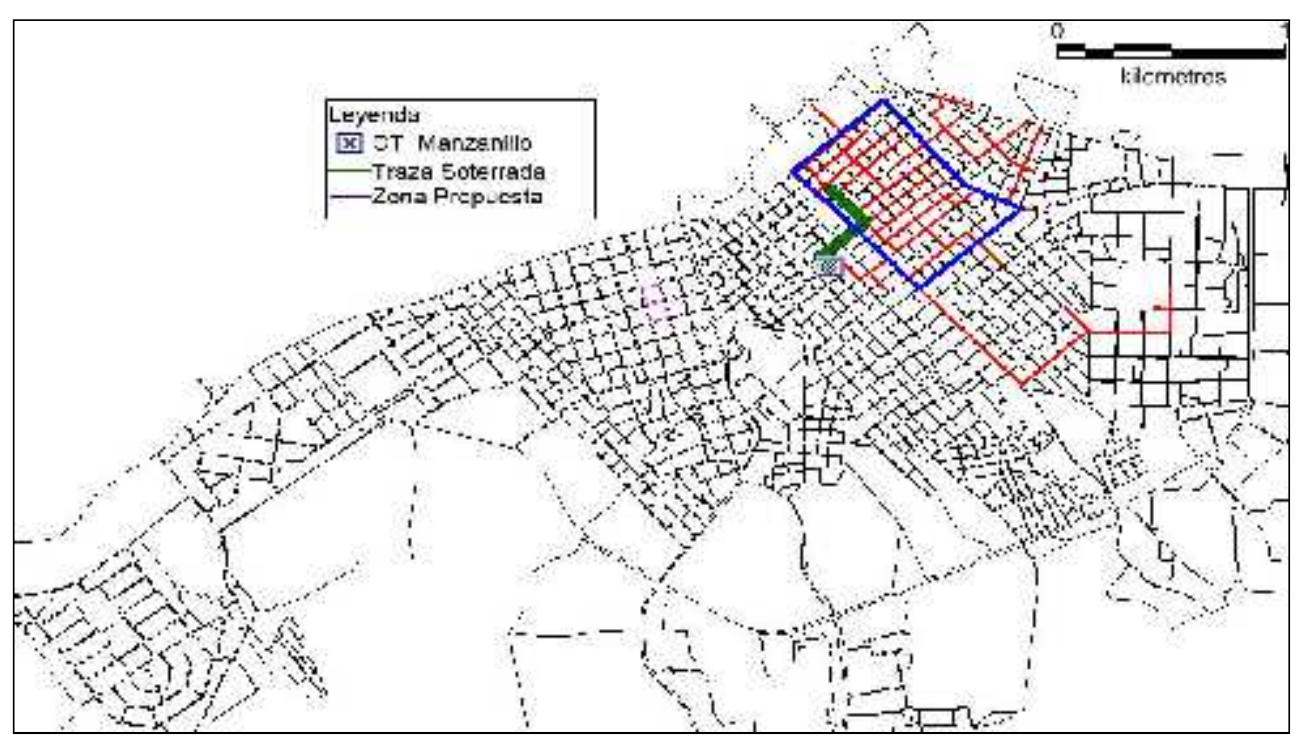

Figura 1: Región del Centro Histórico de la ciudad de Manzanillo

Con estos datos se procedió a realizar la propuesta de red de acceso óptico, la reutilización de la OLT y mejoramiento de algunos equipos teniendo en cuenta las proyecciones futuras de brindar el servicio de internet en las casas.

\section{Propuesta del proyecto}

Los usuarios residenciales, 2159 viviendas, demandan como mínimo 2 Mbps por vivienda, lo que constituye 4318 Mbps para el sector residencial. Las 172 entidades ubicadas en el área demandan hasta 8 Mbps/Usuario, 1376 Mbps para el sector no residencial.

La demanda total, para el sector residencial y no residencial, es de $5694 \mathrm{Mbps}$. Al tener en cuenta las necesidades de velocidad de transmisión, la ubicación geográfica de todos los usuarios y la reutilización de la red de cobre existente, se elige la variante FTTC, con GPON y ADSL2+ como solución de acceso para el escenario objeto de estudio.

Se propone la utilización de la OLT MA5600T del proveedor de equipos de telecomunicaciones Huawei, pues ya está en funcionamiento en el Centro Telefónico. Este equipo emplea hasta 16 tarjetas de 8 puertos GPON. Cada uno de ellos puede servir hasta 64 ONU/MDU, entrega $2.4 \mathrm{Gbps}$ en bajada y recibe hasta 1.2 Gbps en subida. En este sentido, la MDU MA5616 de Huawei puede dar servicio hasta 128 usuarios ADSL2+. 
Rev. SINAPSIS, Edición № 9, Vol. 2, Diciembre 2016

ISSN 1390 - 9770

En la Figura 2 se presenta el diseño de la red de distribución óptica. La OLT se ubica en el Centro Telefónico de Manzanillo, para aprovechar el soterrado existente para el servicio en la zona de estudio.

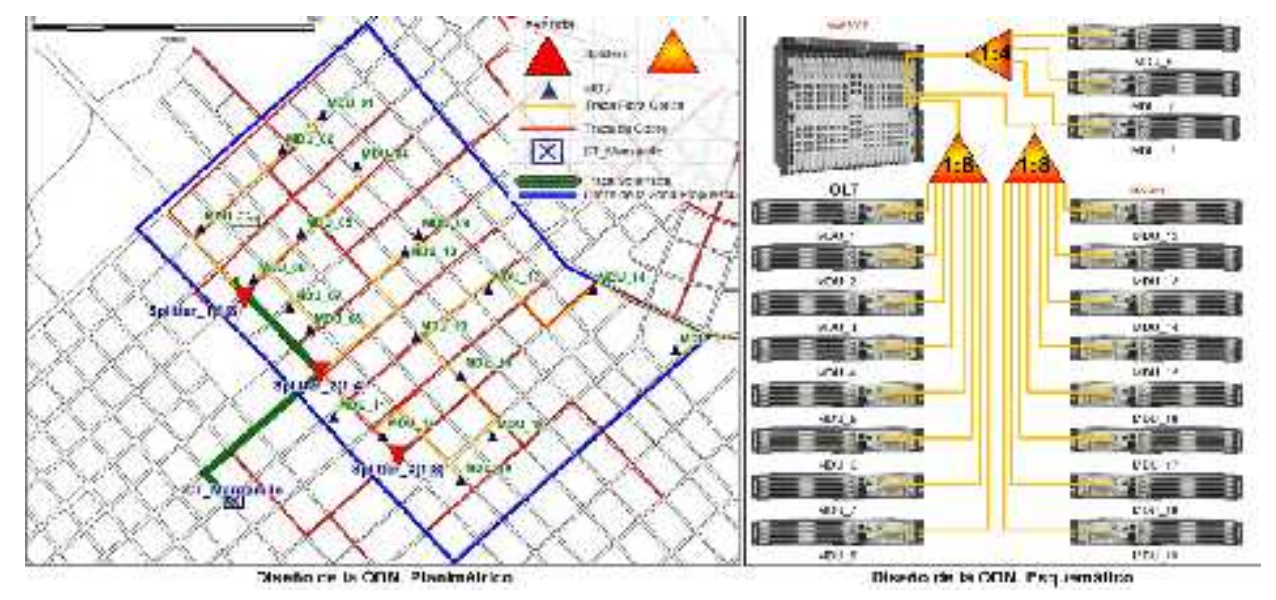

Figura 2: Red de acceso propuesta

Se emplea un solo nivel de divisores ópticos y se ubican los 2 splitters de 1:8 y el splitter de 1:4 en los sitios señalados con los triángulos rojos. Se propone ubicar las 19 MDU en los sitios señalados con triángulos verdes. Para la interconexión de las MDU se utiliza la traza de fibra óptica a partir de la localización de los divisores ópticos pasivos. Se reutiliza, en la medida de lo posible, el cobre existente y en buen estado, interviniendo los empalmes más cercanos a los locales donde se instalen las 19 MDU.

A continuación, se presenta el análisis de presupuesto de potencia del sistema de fibra óptica representado en la Figura 3 (Rodríguez Torres \& Verdecia Peña, 2016). Además, se tuvo en cuenta la topología de la red de prueba de campo desplegada en Cayo Coco, provincia de Ciego de Ávila, Cuba (Rojas-Vargas, 2015).

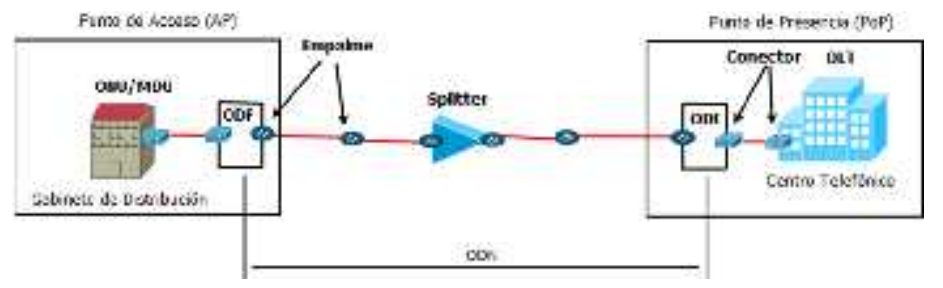

Figura 3. Componentes de la red de acceso óptica

En el cálculo del enlace se consideran las pérdidas introducidas por los conectores, empalmes, las características de la fibra óptica (ITU-T, 2009), divisores ópticos pasivos y un margen de seguridad. 
Rev. SINAPSIS, Edición № 9, Vol. 2, Diciembre 2016

El enlace GPON debe satisfacer el valor de presupuesto de potencia de $28 \mathrm{~dB}$ con láser clase $\mathrm{B}+$, soportada por los equipos ONU/MDU y la OLT propuestos. En la investigación se efectúa el cálculo de atenuación total (AT) para el enlace de mayor longitud, pues constituye el caso más crítico. La AT se calcula según la ecuación 1.

$\mathrm{AT}=\mathrm{L}^{*}(\alpha \mathrm{FO}+\alpha \mathrm{env} \cdot \mathrm{FO})+\alpha \mathrm{E}^{*} \mathrm{NE}+\alpha \mathrm{C}^{*} \mathrm{NC}+\alpha \mathrm{POS}+\mathrm{MS}$

Donde:

L: longitud del enlace $(\mathrm{km})$

$\alpha \mathrm{FO}$ : coeficiente de atenuación de la fibra óptica G.652D (dB)

$\alpha e n v . F O$ : coeficiente de atenuación por envejecimiento de la fibra $(\mathrm{dB} / \mathrm{km})$

$\alpha \mathrm{E}$ : pérdidas en los empalmes (dB)

NE: número de empalmes

$\alpha \mathrm{C}$ : pérdidas en los conectores (dB)

NC: número de conectores

$\alpha$ POS: pérdidas en el splitter (dB)

MS: margen de seguridad $(\mathrm{dB})$

En el cálculo de enlace se asumieron varios valores teóricos de pérdidas introducidas por los componentes del enlace óptico (Farooq, Bashir, Tasneem, Saboor, \& Rauf, 2015). Se empleó el cable de fibra óptica monomodo, coeficiente de atenuación de la fibra G.652D de $0.4 \mathrm{~dB}(1310 \mathrm{~nm}), 0.3 \mathrm{~dB}(1490 \mathrm{~nm})$, coeficiente de atenuación por envejecimiento de $0.05 \mathrm{~dB} / \mathrm{km}$, atenuación por empalmes de $0.1 \mathrm{~dB}$, por conectores de $0.2 \mathrm{~dB}$, atenuación del splitter 1:4 de $7.3 \mathrm{~dB}$ y del splitter 1:8 de $10.4 \mathrm{~dB}$ y el margen de seguridad de $3 \mathrm{~dB}$. Se realizó el análisis para el enlace de mayor longitud de $2 \mathrm{~km}$ que sigue la ruta de postes existentes. Se consideró en el cálculo 4 conectores, 8 empalmes (incluye 2 de reserva) y el splitter 1:8.

En sentido descendente $(\lambda=1490 \mathrm{~nm})$ :

$\mathrm{AT}=2 *(0.3+0.05)+0.1 * 8+0.2 * 4+10.4+3=15.7 \mathrm{~dB}$

En sentido ascendente $(\lambda=1310 \mathrm{~nm})$ :

$\mathrm{AT}=2 *(0.4+0.05)+0.1 * 8+0.2 * 4+10.4+3=15.9 \mathrm{~dB}$ 


\section{Rev. SINAPSIS, Edición № 9, Vol. 2, Diciembre 2016}

Por tanto, el valor obtenido de la atenuación total máxima permisible es menor que el presupuesto óptico de potencia de $28 \mathrm{~dB}$ (clase $\mathrm{B}+$ ), por lo que se cumple con los requerimientos del enlace GPON.

\section{Discusión}

En la actualidad, se hace necesario realizar el despliegue de soluciones de red de acceso óptico asociadas al proceso de informatización de la sociedad y a las redes priorizadas de la salud y la educación, así como para redes vinculadas a la producción de software, centros científicos, universidades y hogares. (Rodríguez Torres \& Verdecia Peña, 2016)

Las tecnologías de línea de abonado digital permiten la transmisión de información digital a través de la red telefónica (Zhao et al., 2013). Ellas ofrecen una mayor razón de transferencia sobre el par de cobre, sin amplificadores ni repetidores de señal a lo largo de la ruta del cable, entre la conexión del usuario y el primer nodo de la red. Las xDSL surgieron con el objetivo de reutilizar la infraestructura en explotación y aprovecharla de manera más eficiente. (Gaona, 2007)

Las redes FTTx posibilitan la eliminación de los cuellos de botella existentes en la última milla de las redes de acceso basadas en pares de cobre o cable coaxial. Para ello, se recurre a la sustitución total o parcial del bucle local por fibra, de manera que se disponga de un medio de transmisión de alta capacidad hasta el usuario final.(Abreu, M., Castagna, A., Cristiani, P., Zunino, P., Roldós, E., \& Sandler, G, 2009) Específicamente, se obtuvo que con la red FTTC se conecta la fibra hasta un gabinete que posee concentradores multiservicios cercanos al usuario y se aprovecha el resto de la red de cobre existente, a través del empleo de tecnologías xDSL, pues se garantizan los requerimientos del alcance físico del enlace.

Las tecnologías PON dan solución a la problemática de la última milla y constituyen una alternativa para los operadores de redes de telecomunicaciones por las significativas ventajas que ofrecen (Rosabal-Montero, 2011): minimizan el despliegue de fibra óptica en el bucle local al poder utilizar topologías punto a multipunto mucho más eficientes que las topologías punto a punto. Dichas redes simplifican la densidad del equipamiento en la oficina central, reduciendo el consumo, elevan la calidad del servicio y simplifican el mantenimiento de la red. Además, se sustituye el tramo de cable de cobre por fibra óptica monomodo y los divisores eléctricos por divisores ópticos pasivos. De esta 
Rev. SINAPSIS, Edición №9, Vol. 2, Diciembre 2016

manera, se eliminan todos los componentes activos en la OSP y se minimiza el planeamiento de la red, lo cual contribuye a la reducción de la inversión o gasto de capital (CAPEX, Capital Expenditure) y de operaciones (OPEX, Operational Expenditure) (Wong, 2012), al formar una económica red de acceso hasta el usuario final.

La tecnología GPON elegida posibilita la implementación de una red flexible de acceso sobre fibra óptica (OAN, Optical Access Network) capaz de soportar los requisitos de banda ancha de los servicios de usuarios residenciales y no residenciales. Ella permite la convergencia de los servicios de telecomunicaciones sobre una única infraestructura de red (Millán, 2008), lo que se evidencia en una notable reducción de los costos para ETECSA, lo cual posibilita el establecimiento de tarifas económicas para los abonados por nuevos servicios que requieren mejores recursos de red, como Internet de alta velocidad, juegos en red, teletrabajo, entre otros.

La combinación de una OLT MA5600T, que alimente a varias MDU MA5616, es una configuración típica para implementar una variante FTTC + xDSL (Ramos, 2016), con la cual se garantice el acceso a la banda ancha en la región analizada. Se necesitan 3 puertos GPON de una OLT MA5600T para cubrir la velocidad de conexión demandada y 19 MDU MA5616 para dar acceso a las 2159 viviendas y 172 entidades de la zona.

El diseño de la red de acceso óptica cumple con los requerimientos de presupuesto de potencia del enlace GPON con valores de pérdidas inferiores a $28 \mathrm{~dB}$ en sentido descendente y ascendente para el caso más crítico de mayor longitud de la ODN. Los valores obtenidos evidencian que el sistema queda listo para la inserción de un segundo nivel de splitter para la futura expansión de la red óptica pasiva. (López, M., Moschim, E. \& Rudge, F., 2009)

Se recomienda que esta investigación se utilice como material de estudio para estudiantes universitarios de la carrera de telecomunicaciones y todas las ramas asociadas. El mismo permite ampliar la visión para la realización de propuestas de red de acceso óptico.

\section{Conclusiones}




\section{Rev. SINAPSIS, Edición № 9, Vol. 2, Diciembre 2016}

Se concluye que el proyecto ayudará en primer lugar la instalación del servicio de banda ancha hacia los hogares del Centro Histórico de Manzanillo y en segundo lugar que toda la implementación y puesta en práctica del proyecto servirá de instrumento de estudio para los estudiantes de la carrera de Telecomunicaciones en el país ya que facilitará la demostración práctica de la red de acceso óptica.

\section{Bibliografía}

1. Abreu, M., Castagna, A., Cristiani, P., Zunino, P., Roldós, E., \& Sandler, G. (2009). Carácterísticas generales de una red de fibra óptica al hogar (ftth). Memoria de Trabajos de Difusión Científica Y Técnica, 7, 38-46. Retrieved from http://www.um.edu.uy/_upload/_descarga/web_descarga_179_Caractersticasgener alesredfibrapticaalhogarFTTH.-VVAA.pdf

2. Ansari, N., \& Zhang, J. (2013). Chapter 2 PON Architectures. Media Access Control and Resource Allocation: For Next Generation Passive Optical Networks (pp. 11-22). Springer. doi:10.1007/978-1-4614-3939-4

3. Carballo-González, C., Conde del Oso, L. E., \& Anías-Calderón, C. (2016). Propuesta de soluciones de acceso para la introducción por ETECSA de los servicios de banda ancha en Cuba. Informática 2016 (pp. 1-10).

4. FTTH Council Europe. (2012). FTTH Handbook. Retrieved from http://www.ftthcouncil.eu/documents/Publications/FTTH-Handbook_2014V6.0.pdf

5. Farooq, U., Bashir, S., Tasneem, T., Saboor, A., \& Rauf, A. (2015). Migration from Copper to Fiber Access Network using Passive Optical Network for Green and Dry Field Areas of Pakistan. International Journal of Soft Computing and Engineering (IJSCE), 5(4), 118-128.

6. Gaona, H. (2007). XDSL en el lazo de abonado. Revista Digital Universitaria, 8 (10), 1-12

7. ITU-T. (2008). Gigabit-capable passive optical networks (GPON): General characteristics. Recommendation ITU-T G.984.1. Retrieved from https://www.itu.int/rec/T-REC-G.984.1-200803-I/en

8. ITU-T. (2009). Characteristics of a single-mode optical fibre and cable.

Recommendation ITU-T G.652. Retrieved from http://www.itu.int/rec/T-RECG.652-200911-I/en

9. ITU-T. (2014a). The State of Broadband 2014: broadband for all. Retrieved May 10, 2016, a from http://www.broadbandcommission.org/documents/reports/bbannualreport2014.pdf 
Rev. SINAPSIS, Edición № 9, Vol. 2, Diciembre 2016

10. ITU-T. (2014b). Connect 2020: Setting a global agenda for the ICT sector, 1-2. Retrieved from

http://www.itu.int/en/plenipotentiary/2014/newsroom/Documents/backgrounders/p p14-backgrounder-connect-2020-es.pdf

11. Koonen, T. (2006). Fiber to the Home/Fiber to the Premises: What, Where, and When? Proceedings of the IEEE, 94(5), 911-934. oi:10.1109/JPROC.2006.873435

12. López, M., Moschim, E. \& Rudge, F. (2009). Estudio comparativo de redes GPON y EPON. Revista Scientia et Technica ISSN 0122-1701. XV (41), 1-6.

13. Lafata, P. (2013). Examination of Multiplexing VDSL2 over ADSL2+ Line. Elektronika ir Elektrotechnika, 19(8), 123-127. doi:10.5755/j01.eee.19.8.3116

14. Mahloo, M. (2015). Transport Solutions for Future Broadband Access Networks. KTH Royal Institute of Technology, Stockholm, Sweden. Retrieved from https://www.diva-portal.org/smash/get/diva2:796654/FULLTEXT01.pdf

15. Millán, R. (2008). GPON (Gigabit Passive Optical Network). Revista Bit 166, 1-5.

16. Ramos, M. (2016). Diseño de una red de acceso FTTH-GPON para una urbanización en la parroquia Cumbayá con servicios triple play. Tesis de titulación en Ingeniería en Redes y Telecomunicaciones. Facultad de Ingeniería y Ciencias Agropecuarias, UDLE.

17. Rodríguez Torres, M., \& Verdecia Peña, R. (2016). Propuesta de Comunicación con Tecnología Óptica Pasiva en el Reparto "Carlos Manuel de Céspedes" de Bayamo, Cuba: opción de estudio para los universitarios. SINAPSIS, 8(1), 1-11.

18. Rojas-Vargas, A. G. (2015). Impacto y explotación de las redes GPON en Cuba. XVI Convención de Ingeniería Eléctrica (CIE-2015).

19. Rosabal-Montero, D. (2011). Tecnologías de acceso sobre redes ópticas pasivas. Serie Científica de la Universidad de las Ciencias Informáticas, 4(3), 1-12. Retrieved from publicaciones.uci.cu/index.php/SC/article/view/376/381

20. UIT. (2013). Banda ancha. Foro Mundial de Política de las Telecomunicaciones/TIC. Retrieved June 10, 2016, from http://www.itu.int/en/wtpf-13/Documents/backgrounder-wtpf-13-broadband-es.pdf

21. Wong, E. (2012). Next-Generation Broadband Access Networks and Technologies. Journal of Lightwave Technology, 30(4), 597-608. doi:10.1109/JLT.2011.2177960

22. Zhao, R., Fischer, W., Aker, E., \& Rigby, P. (2013). Broadband Access Technologies. White Paper, 6-7. Retrieved from http://www.ftthcouncil.eu/documents/Publications/DandO_White_Paper_2_2013_ Final.pdf 